\title{
Notas florísticas sobre algunas gramíneas de Flora iberica
}

\author{
Josefa López ${ }^{1}$, Ana Ortega-Olivencia ${ }^{1}$, Tomás Rodríguez-Riaño ${ }^{1}$ \& Juan Antonio Devesa ${ }^{2}$ \\ ${ }^{1}$ Área de Botánica, Facultad de Ciencias, Universidad de Extremadura, Avenida de Elvas, s.n., 06006 Badajoz, España. \\ Departamento de Botánica, Ecología y Fisiología Vegetal, Facultad de Ciencias, Universidad de Córdoba, Campus de \\ Rabanales, Edificio José Celestino Mutis, Ctra. de Madrid km. 396 A, 14014 Córdoba, España.
}

\section{Correspondencia}

A. Ortega-Olivencia e-mail: aortega@unex.es

Recibido: 20 junio 2018

Aceptado: 20 septiembre 2018

Publicado on-line: septiembre 2018

\section{Floristic notes on some grasses of Flora iberica}

Palabras clave: Corología, Flora iberica, Gramineae, Península Ibérica, Poaceae, Pooideae.

Key words: Chorology, Flora iberica, Gramineae, Iberian Peninsula, Poaceae, Pooideae.
Con motivo del estudio de los géneros Glyceria R. Br., Ctenopsis De Not., Psilurus Trin., Parapholis C.E. Hubb. y Apera Adans. para el volumen XIX de Flora iberica, se han revisado unos 1860 pliegos conservados en los herbarios BC, COI, GDAGDAC, HSS, HUAL, JAEN, LEB, LISU, MA, MAF, MGC, SALA-SALAF, SANT, SEV, UNEX y VAL. Fruto de ello ha sido la elaboración de las síntesis taxonómicas de estos géneros, que verán la luz en su día, y en las que hay algunas novedades corológicas de importancia que se dan a conocer en este trabajo. Algunas implican la ampliación de las áreas de distribución de algunos taxones y otras su modificación al haber sido estudiados los materiales testigo que soportaban las citas bibliográficas.

\section{Glyceria R. Br.}

El género comprende 48 especies nativas de Eurasia, $\mathrm{N}$ de África y América (Soreng et al., 2017), de las que solo cuatro están presentes en la Península lbérica: Glyceria fluitans (L.) R.Br., G. declinata Breb., G. notata Chevall. y G. spicata Biv. ex Guss.

Glyceria striata (Lam.) Hitchc. in Proc. Biol. Soc. Wash. 41: 157 (1928)

Poa striata Lam., Tabl. Encycl. 1: 183 (1791)

El taxón, que fue descrito de Estados Unidos de América ("E Virginia, Carol."), extiende su área de distribución también por el $\mathrm{S}$ de Canadá y $\mathrm{N}$ de Méjico (Tzvelev, 2006). Ha sido introducido en Europa, incluyendo Ucrania y Rusia (Holub, 1980; Valdés \& Scholz, 2009), aunque actualmente se discute sobre si debe considerarse casual o naturalizado en este continente (Valdés \& Scholz,
2009). Su presencia en el territorio de Flora iberica fue señalada por Vázquez \& Cabeza (2009) para las inmediaciones de Berlanga (Badajoz), pero el estudio del material testigo (HSS 33348) ha puesto de manifiesto que se trata en realidad de Poa infirma Kunth. Con los datos disponibles, y tras no haberse detectado material alguno que se identifique con Glyceria striata, se descarta esta especie del ámbito de Flora iberica.

\section{Ctenopsis De Not.}

Comprende 4 especies, con representación en la Región Mediterránea y el W de Asia (Watson \& Dallwitz, 1992), que algunos autores reunieron en una sección dentro del género Vulpia C.C. Gmel. (Clayton \& Renvoize, 1986); más modernamente, estudios de filogenia con marcadores moleculares emplazan Ctenopsis dentro del género Festuca L. (Soreng et al., 2017). Con independencia de ello, en su concepción clásica está representado en la Península lbérica solo por Ctenopsis delicatula (Lag.) Paunero y C. gypsophila (Hack.) Paunero.

Ctenopsis delicatula (Lag.) Paunero in Anales Inst. Bot. Cavanilles 21: 365 (1964)

Festuca delicatula Lag. in Varied. Ci. 2(4): 39 (1805)

Moreno-Saiz \& Sáinz-Ollero (1992) habían indicado Ctenopsis delicatula en Douro Litoral basándose en una recolección de Gonçalo Sampaio ("Villa Nova de Gaya, no Areinho") que, a su vez, había citado Henriques (1903, sub Festuca delicatula), cita que no habían podido confirmar. Sin duda, la planta recolectada por G. Sampaio en "Gaya, Areinho d'Avintes en mayo de 1898 (COI 00011283, sub Festuca delicatula) pertenece a $C$. 
delicatula, la cual debe ser rara en esta provincia, ya que ni Coutinho (1939) ni Franco \& RochaAlfonso (1998) la indican de esta provincia en sus respectivas floras; tampoco Sequeira et al. (2011) y Romero-Zarco (2015), y en la Flora de Portugal Interactiva (Flora-On, 2014) no aparece ninguna localidad del taxón en Douro Litoral. Por el contrario, su presencia en la provincia portuguesa de Alto Alentejo debe ser rechazada. Las citas que la apoyaban (Moreno-Saiz \& Sáinz-Ollero, 1992, recogida también en Anthos, 2012) están basadas en material procedente de Nisa (MAF 82621) y Portalegre (LEB 5041), pero el estudio de dichos materiales testigo ha revelado en ambos casos que se trata de Chaetopogon fasciculatus (Link) Hayek. Revisadas las bases de datos (Flora-On, 2014) y toda la bibliografía sobre el género relativa a Portugal (Coutinho, 1939; Franco \& RochaAlfonso, 1998; Sequeira et al., 2011; RomeroZarco, 2015), así como el material conservado en los herbarios portugueses de Lisboa (LISU, LISE) y Coimbra (COI), no se han encontrado citas ni materiales de esta especie en Alto Alentejo.

Se trata de un terófito endémico del $\mathrm{CW}$ de España (Av Ba Cc CR Gu M Sa Sg To Va Z Za) y el $\mathrm{N}$ de Portugal [BA DL (Mi) TM], que habita en pastizales acidófilos de dehesas, claros de melojar, cunetas, taludes y campos incultos, sobre todo en suelos arenosos o pizarrosos, entre 700 y 1400 m, más escasa en suelos salobres o calcáreos.

Ctenopsis gypsophila (Hack.) Paunero in Anales Inst. Bot. Cavanilles 21: 368 (1964)

Festuca gypsophyla Hack. in Oesterr. Bot. Z. 27: 47 (1877)

Material referenciado. PORTUGAL. Ribatejo.

Pr. Constancia, matos de cocciferetum, 1-V-1955, M. Beliz et al. (UNEX 1820).

Terófito cuya área de distribución se extiende por la Península Ibérica y el W de Sicilia, propio de pastizales xerofíticos, en cerros yesosos y suelos salobres, desde los 600 a los $1300 \mathrm{~m}$. Hasta ahora no había sido indicado para la flora de Portugal (Coutinho, 1939; Franco \& RochaAlfonso, 1998; Sequeira et al., 2011; RomeroZarco, 2015; Flora-On, 2014), pero del herbario de la Universidad de Extremadura se ha estudiado un pliego (UNEX 1820) con material de Ctenopsis gypsophila recolectado en la provincia de Ribatejo, que constituye la primera cita de la especie para Portugal.

El área de distribución del taxón en España peninsular es bien conocida y se extiende por algunas provincias del C, CW y SE [(Av) Ab Al CR Cu Gr (Gu) J M To Va (Za)]. De esta distribución se ha excluido la provincia de Cáceres, donde había sido indicada por Galicia et al. (1993) para Baños de Montemayor, pues el material testigo estudiado (MACB 36561; MA 491227), recolectado por Arturo Caballero el 15 de mayo de 1949, se corresponde inequívocamente con Micropyrum patens (Brot.) Pilg. Por otro lado, las apetencias ecológicas de la especie hacen muy difícil su presencia en esta provincia -y en la región- y, de hecho, ni ha sido citada en estudios previos (Paunero, 1964; Devesa, 1991; Devesa \& Vázquez, 1992; Devesa, 1995; Romero-Zarco, 2015) ni se ha encontrado testimonio alguno de herbario.

\section{Psilurus Trin.}

Género monotípico cuya única especie se distribuye por la Región Mediterránea, aunque alcanza por el este el SW y el S de Asia -hasta Pakistán-, y ha sido introducida en otras zonas de la Tierra (Watson \& Dallwitz, 1992). Al igual que los géneros anteriores, los estudios de filogenia emplazan a Psilurus dentro del género Festuca L. (Soreng et al., 2017).

Psilurus incurvus (Gouan) Schinz \& Thell. in Vierteljahrsschr. Naturf. Ges. Zürich 58: 40 (1913)

Nardus incurva Gouan, Hortus Monsp:: 33 (1762)

Material referenciado. PORTUGAL. Beira Litoral. Pinhal de Marroes, Coimbra, V-1879, A. Moller (COI 12189). Douro Litoral. Porto, Foz-Fua, VI-1914, G. Sampaio (MA 14775). Estremadura. Alfeite, margem esquerda do Tejo, VI-1892, J. Daveau (COI 12190). Lisboa, Porares c. de Freixo de Espada á Cinta entre Qta. da Malhadinha ea ribeira do Mosteiro, 2-V-1946, G. Barbosa \& F. Garcia (MA 412331).

Se trata de una especie indiferente edáfica, que habita en pastizales terofíticos efímeros, sobre suelos arenosos o pedregales, desde el nivel del mar hasta 1510(1600) m. De acuerdo con el material estudiado y con la información contenida en las bases de datos Anthos (2012) y Flora-On (2014), así como de la monografía de RomeroZarco (2015) y diversas floras portuguesas (v.g. Coutinho, 1939; Franco \& Rocha-Afonso, 1998), su área de distribución en el territorio de Flora iberica se extiende por buena parte de la Península Ibérica y Baleares [Esp.: A (Ab) Al B Ba (Bu) Cc Co CR (Cs) Ge Gr (Gu) H (Hu) J L (Lo) (Lu) M (Ma) (Na) PM [(lb) (MII)] (Po) Sa Se Sg T (Te) (To) V Vi (Z) Za. Port.: AAI Ag BA BAI BB BL DL E (R) (TM)], distribución que incluye las provincias portuguesas de Beira Alta, Beira Litoral, Douro Litoral y Estremadura, indicadas aquí por vez primera.

En el estudio morfológico efectuado se ha 
detectado gran variabilidad en el tamaño de las anteras, que varía de 0,25 a 1,9(2,5) mm, lo que no solo se ha observado entre individuos de poblaciones diferentes, sino también en el seno de una misma población, e incluso en una misma inflorescencia. En efecto, en algunas poblaciones se ha detectado un tamaño decreciente en las anteras desde el ápice de la espiga hasta su base (v.gr., en material de Salamanca, MA 371108, y de Badajoz, MA 718023), mientras que en otros se ha observado la coexistencia de flores con anteras de tamaño pequeño y grande entremezcladas a lo largo de una misma espiga (v.gr., provincia de Jaén, MA 618900). También, hay plantas en las que las flores de todas las espiguillas de una espiga en antesis tienen anteras pequeñas $(0,25-0,6 \mathrm{~mm})$, y otras en las que todas las anteras son grandes (1-1,9 mm). No parece haber una relación entre el tamaño de la antera y la distribución geográfica, las apetencias ecológicas o la fenología de la floración en el ámbito de esta flora, y no se sabe qué factores son los responsables de esta variación.

Parapholis C.E. Hubb.

Comprende 6 especies y está representado en el N y W de Europa, Región Mediterránea y en el SW y S de Asia (Watson \& Dallwitz, 1992). De ellas, solo Parapholis gracilis Bor, endémica de Iraq, no está presente en la Península Ibérica y Baleares.

Parapholis marginata Runemark in Bot. Not. 115: 8, 14, t. 3D (1962)

Material referenciado. ESPAÑA. Huelva. EI Rompido, flecha litoral, 2008, ambiente salobre y de marisma, 4 m, 29S666E 4120N, S. Muñoz (SEV 273910).

Especie propia de pastizales terofíticos desarrollados en saladares, marismas y pequeñas depresiones postdunares, en suelos arenosos por debajo de los $5 \mathrm{~m}$ sobre el nivel del mar. En el ámbito de Flora iberica se conocía solo del este de España (provincias de Alicante y Murcia) y Baleares (Mallorca y Formentera), por lo que la cita aportada aquí amplía su área de distribución a Andalucía occidental (provincia de Huelva).

La especie incluye plantas pequeñas y robustas, con espigas agrupadas, glumas con quilla claramente alada y anteras hasta de $0,9 \mathrm{~mm}$. Por su hábito puede confundirse con Parapholis incurva (L.) C.E. Hubb., de la que se diferencia por la presencia de las glumas aladas y espigas agrupadas.

Parapholis strigosa (Dumort.) C.E. Hubb. in Blumea, Suppl. 3: 14 (1946)

Lepturus strigosus Dumort., Observ. Gramin.
Belg.: 146 (1824)

Especie propia de los pastizales terofíticos halófilos que se desarrollan en suelos arenosos de saladares y marismas, entre el nivel del mar y $690 \mathrm{~m}$. En la Península lbérica su distribución se restringe al N y NO (Esp.: Bi C Le Lo Lu O P Po S SS Za. Port.: BL), si bien había sido indicada también para las provincias andaluzas de Cádiz (Sierra de Líjar, SEV 59041 y MGC 11298 duplicado del anterior-; Aparicio \& Cabezudo, 1982) y Málaga (Fuentepiedra, Runemark, 1962), y para la Comunidad Valenciana (Torreblanca, Prat Cabanes, provincia de Castellón, VAL 26347; Tirado et al., 1994). Sin embargo, el estudio del material testigo de estas citas pone de manifiesto que deben ser rechazadas por erróneas. Las plantas castellonenses y las de Andalucía occidental (para la que Talavera -1987- no reconoce la presencia del taxón) que nosotros hemos estudiado se corresponden en realidad con P. pycnantha (Hack.) C.E. Hubb. Probablemente, el error de identificación de estos pliegos sea debido a que los individuos que los integran presentan flores con anteras mal conformadas -estériles-, más pequeñas de lo normal, situándose el tamaño de estas en el límite de separación de ambas especies. Sin embargo, la morfología de las glumas permite identificarlas como P. pycnantha, una especie que se distribuye por el S y E de España, y Baleares [Ca Cs (H) Ma $\mathrm{PM}[\mathrm{Mn}] \mathrm{Se} \mathrm{V}]$. Respecto de la cita malagueña, que Romero-García (2009) indica que ha de ser confirmada, casi con toda seguridad debe ser referida también a $P$. pycnantha por los mismos motivos.

\section{Apera Adans.}

El género comprende 5 especies (Soreng et al., 2017), de las que dos están presentes en el territorio de Flora iberica: Apera spicata-venti (L.) Beauv. y A. interrupta (L.) Beauv.

Apera interrupta (L.) Beauv., Ess. Agrostogr. 31: 151 (1812)

Agrostis interrupta L., Syst. Nat. ed. 10, 2: 872 (1759)

Material referenciado. ESPAÑA. Gerona. Cerdagne, Sareja à Tudó, 29-VII-1918, champs, 1400 m, F. Sennen, Plantes d'Espagne (MA 7005, 1 \& 2). Logroño. 3-VII, I. Zubia (MA 6987, 1 \& 2; SEV 6100).

Especie anual cuya área de distribución se extiende por el W, C, N y $\mathrm{S}$ de Europa y que alcanza el SE de Rusia, SW de Asia, así como el $\mathrm{N}$ de África; está naturalizada en Argentina y se la conoce como adventicia en Chile (Nicora \& Rúgolo, 1987). En el ámbito de estudio se comporta como 
indiferente edáfica y habita en pastizales sobre suelos arenosos, claros de bosque y márgenes de cultivos, con un rango altitudinal de (700)7501800(2000) m. Se encuentra en la Península Ibérica, sobre todo en la mitad septentrional y oriental [Esp.: (Ab) (Al) Av Bu (CR) (Cu) Ge Gr (Gu) $\mathrm{Hu} J(\mathrm{~L})$ Le Lo M (Ma) (Mu) (O) (Or) Sa Sg So T Te Va (Vi) Z Za. Port.: (DL) (TM)], distribución que incluye por vez primera los territorios de Gerona y Logroño, de los que no existía constancia alguna ni en la base de datos de Anthos (2012) ni en la bibliografía previa consultada (v.gr., Bolòs \& Vigo, 2001; Romero-Zarco, 2015; Font, 2018).

\section{Agradecimientos}

Nuestra sincera gratitud a los conservadores de los herbarios consultados y a los dos revisores por sus sugerencias. Este estudio ha sido subvencionado por el Ministerio de Ciencia e Innovación a través de los proyectos CGL2008-0298-Co3-3, CGL201128613-C03-02 y CGL2014-52787-C8-3-P, todos ellos cofinanciados por FEDER.

\section{Referencias}

Anthos (2012). Sistema de Información sobre las plantas de España. Fundación Biodiversidad-Jardín Botánico de Madrid, CSIC. http://www.anthos.es [consultado el 12-06-2018].

Aparicio, A. \& Cabezudo, B. (1982). Aportaciones al conocimiento florístico de la provincia de Cádiz (Sierra de Líjar). Anales del Jardín Botánico de Madrid, 38(2), 477-483.

Bolòs, O. \& Vigo, J. (2001). Flora dels Països Catalans 4. Barcelona: Editorial Barcino.

Clayton, W.D. \& Renvoize, S.A. (1986). Genera Graminum. London: Kew Bulletin Additional Series XIII.

Coutinho, A.X.P. (1939). Flora de Portugal, $2^{\mathrm{a}}$ edição. Lisboa: Irmãos Bertrand, Lda.

Devesa, J.A. (1991). Ctenopsis De Not. In J.A. Devesa (Ed.), Las Gramíneas de Extremadura (pp. 56-58). Badajoz: Servicio de Publicaciones-UNEX.

Devesa, J.A. (1995). Ctenopsis De Not. In J.A. Devesa (Ed.), Vegetación y Flora de Extremadura (pp. 594). Badajoz: Universitas Editorial.

Devesa, J.A. \& Vázquez, F.M. (1992). Ctenopsis De Not. In J.A. Devesa (Ed.), Anatomía foliar y palinología de las gramíneas extremeñas (pp. 68-69). Badajoz: Servicio de Publicaciones-UNEX.

Font, X. (2018). Mòdul Flora i Vegetació. Banc de Dades de Biodiversitat de Catalunya. Generalitat de Catalunya i Universitat de Barcelona. http://biodiver. bio.ub.es/biocat/homepage.html [consultado el 12-062018].

Flora-On (2014). Flora de Portugal Interactiva. Sociedade Portuguesa de Botânica. http://www.floraon.pt [consultado el 12-06-2018].

Franco, J.A. \& Rocha-Afonso, M.L. (1998). Nova Flora de Portugal III Gramineae, fasc. II. Lisboa: Escolar Editora.

Galicia, D., Moreno-Rivero, L. \& Moreno-Saiz, J.C. (1993). Cartografía corológica ibérica: Aportaciones 46-48. Botanica Complutensis, 18, 322-328.

Henriques, J. (1903). Subsidio para o conhecimento da Flora Portugueza. Boletim da Sociedade Broteriana 20: VII-XV, 1-183.

Holub, J. (1980). Glyceria R.Br. In T.G. Tutin et al. (eds.), Flora Europaea 5 (pp. 179-181). Cambridge: Cambridge University Press.

Moreno-Saiz, J.C. \& Sáinz-Ollero, H. (1992). Atlas corológico de las monocotiledóneas endémicas de la Península Ibérica e Islas Baleares. Madrid: Colección Técnica, Mapa, ICONA.

Nicora, E.G. \& Rúgolo, Z.E. (1987). Los géneros de gramíneas de América austral. Buenos Aires: Editorial Hemisferio Sur S.A.

Paunero, E. (1964). El género Ctenopsis De Not. en la flora española. Anales del Instituto Botánico Cavanilles, 21(2), 357-386.

Romero-García, A.T. (2009). Parapholis C.E. Hubbard. In G. Blanca et al. (Eds.), Flora Vascular de Andalucía Oriental 1 (pp. 361-362). Sevilla: Consejería de Medio Ambiente, Junta de Andalucía.

Romero-Zarco, C. (2015). Las gramíneas de la Península Ibérica e Islas Baleares. Monografías de Botánica Ibérica 15. Jaca: Jolube Consultor Botánico y Editor.

Runemark, H. (1962). A revision of Parapholis and Monerma in the Mediterranean. Botaniska Notiser, 115(1), 1-17

Sequeira, M., Espírito-Santo, D., Aguiar, C., Capelo, C. \& Honrado, J.J. (Eds.) (2011). Checklist da Flora de Portugal Continental, Açores e Madeira. Lisboa: ALFA.

Soreng, R.J., Peterson, P.M., Romaschenko, K., Davidse, G., Teisher, J.K., Clark, L.G., Barberá, P., Gillespie, L.J. \& Zuloaga, F.O. (2017). A worldwide phylogenetic classification of the Poaceae (Gramineae) II: an update and comparison of two 2015 classifications. Journal of Systematics and Evolution, 55(4), 259-290. https://doi.org/10.1111/jse.12262.

Talavera, S. (1987). Parapholis C.E. Hubbard. In B. Valdés et al. (eds.), Flora Vascular de Andalucía Occidental 3 (pp. 299-301). Barcelona: Ketres Editora S.A.

Tirado, J., Villaescusa, C. \& Aguilella, A. (1994). Fragmenta chorologica occidentalia, 4921-4961. Anales del Jardín Botánico de Madrid, 51(2), 293-295.

Tzvelev, N.N. (2006). Synopsis of the mannagrass genus, Glyceria (Poaceae). Botanischeskii Zhurnal (Moscow and Leningrad), 91(2), 255-276.

Valdés, B. \& Scholz, H.; with contributions from E. von Raab-Straube \& G. Parolly (2009). Poaceae (pro parte majore). Euro+Med Plantbase - the information resource for Euro-Mediterranean plant diversity. http:// ww2.bgbm.org/EuroPlusMed/ [consultado el 12-062018].

Vázquez, F.M. \& Cabeza, M. (2009). Anotaciones corológicas y taxonómicas a la flora en Extremadura. 23. Glyceria striata (Lam.) A.S. Hitch. Folia Botanica Extremadurensis, 4, 64-65.

Watson, L. \& Dallwitz, M.J. (1992). The grass genera of the world. Wallingford: Cab International. 